\section{REPEATED PRE AND POST-SHIFT URINALYSES SHOW KIDNEY DYSFUNCTION AMONG COSTA RICAN SUGARCANE CUTTERS EXPOSED TO HEAT STRESS}

\begin{abstract}
${ }^{1,2}$ Jennfier Crowe, ${ }^{2}$ Maria Nilsson, ${ }^{2}$ Tord Kjellstrom, ${ }^{3}$ Manuel Cerdas, ${ }^{4}$ Richard Johnson, ${ }^{5}$ Catharina Wesseling. ${ }^{1}$ Regional Institute for Studies on Toxic Substances (IRET), Universidad Nacional, Heredia, Costa Rica; ${ }^{2}$ Umea University, Faculty of Medicine, Department of Public Health and Clinical Medicine, Epidemiology and Public Health Sciences, Umea, Sweden; ${ }^{3}$ Department of Nephrology, Hospital México, Heredia, Costa Rica; ${ }^{4}$ Division of Renal Diseases and Hypertension, University of Colorado Anschutz Medical Campus, Colorado, USA; ${ }^{5}$ Institute of Environmental Medicine, Karolinska Institutet, Stockholm, Sweden
\end{abstract}

\subsection{6/oemed-2014-102362.157}

Objectives Sugarcane harvesters in Costa Rica are exposed to heat stress, a likely major risk factor in the Mesoamerican nephropathy epidemic. Routine urinalyses provide important information about kidney function, but have not been reported in detail for this population.

Method Sugarcane cutters $(n=48)$ provided six spot urine samples, pre- and post-workshift for three days during one week in mid-harvest. Chemical analyses with dipstick and microscopic examinations of sediment were performed. Changes over the workday in markers for hydration status and kidney responses to heat stress were evaluated with McNemar test on paired proportions.

Results Preliminary results indicate percentages of workers with specific parameters in at least one morning versus in at least one afternoon sample were with dipstick: $\mathrm{pH} \leq 530 \%$ vs $82 \%$, specific gravity $\geq 1.02530 \%$ vs $51 \%$, proteinuria ( $1+$ and up) $86 \%$ vs $8 \%$, and blood (traces and up) $28 \%$ vs $12 \%$; and in sediment: $>5$ leucocytes $76 \%$ vs $57 \%,>3$ erythrocytes $50 \%$ vs $37 \%$, and casts (granular, leukocyte and some erythrocyte) $75 \%$ vs $39 \%$. Except for erythrocytes in sediment, these differences were statistically significant.

Conclusions Concentration and acidification of urine over the workday indicate insufficient hydration. Positive blood on dipstick may be a sign of low-grade hematuria or mild rhabdomyolysis. Predominance of proteinuria, leucocytes, erythrocytes and casts in morning urine was unexpected but may reflect improvement in glomerular filtration with hydration overnight or, alternatively, effects from vasopressin at night. This study adds to an increasing body of evidence of kidney dysfunction among heat stress exposed sugarcane workers.

\section{INCORPORATING MORE DETAILED EXPOSURE ASSESSMENT WITH QUANTITATIVE ESTIMATES IS ASSESSING THE BURDEN OF OCCUPATIONAL CANCER}

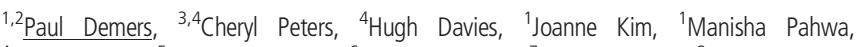
${ }^{4}$ Chris McLeod, ${ }^{5}$ Anne-Marie Nicol, ${ }^{6}$ France Labreche, ${ }^{7}$ Jerome Lavoue, ${ }^{8}$ Sally Hutchings, ${ }^{8}$ Lesley Rushton. ${ }^{1}$ Occupational Cancer Research Centre, Toronto, Canada; ${ }^{2}$ University of Toronto, Toronto, Canada; ${ }^{3}$ CAREX Canada, Vancouver, Canada; ${ }^{4}$ University of British Columbia, Vancouver, Canada; ${ }^{5}$ Simon Fraser University, Vancouver, Canada; ${ }^{6}$ L'Institut de Recherche Robert-Sauvé en Santé Et en Sécurité Du Travail, Montreal, Canada; ${ }^{7}$ Universite de Montreal, Montreal, Canada; ${ }^{8}$ Imperial College, London, UK
}

\subsection{6/oemed-2014-102362.158}

Objectives In recent years, several new burden projects have been initiated with increased methodological sophistication. Previous studies have varied with respect to methods used to identify the prevalence and relevant levels of exposure, but many have relied on CAREX estimates. In this presentation, we will focus on the impact of incorporating more detailed exposure assessments with quantitative estimates as part of the Canadian burden of cancer project.

Method The Canadian exposure estimation process relies on data from CAREX Canada, taking into account industry and occupation at a more detailed level than previously. For many common carcinogens, the Canadian Workplace Exposure Database is used to account for changes in exposure levels over time and quantitative exposure-response relationships from the literature are used to assign relative risks relevant to the mean level of each exposure group. Historical employment trends are based upon census data at multiple time-points with province, sex, industry, and occupation detail.

Results Developing estimates for approximately 300 industries, as well as by occupation, and the need for estimating the age and gender characteristics of predicted cases to estimate economic burden, has also increased the complexity of estimating historic labour force dynamics. Annual labour force data 19762010 is used to attribute age- and tenure-distribution characteristics by province, sex, and industry.

Conclusions Although our main objective was to increase the validity of the burden estimation process, the more detailed exposure estimates allow us to calculated cancer burden for much more specific industry sectors and occupations, allowing for detailed risk reduction strategies.

\section{EXPOSURE-RELATED COMORBIDITIES IN OCCUPATIONAL EPIDEMIOLOGY: SHOULD WE BE USING COMPETING RISKS AND MULTISTATE MODELS?}

Katie Applebaum. George Washington University, School of Public Health and Health Services, Washington, DC, USA

\subsection{6/oemed-2014-102362.159}

Objectives In occupational epidemiology, we often rely on analytical models that look at the relationship between one exposure and one disease. However, the exposure may be related to more than one outcome at the target site (e.g., pulmonary diseases: pneumoconiosis, byssinosis, chronic obstructive pulmonary disease, or lung cancer), and one of these diseases may influence the occurrence of another. Cox proportional hazards regression models using Kaplan-Meier (KM)-based estimates may not be appropriate due to violating the model's non-informative censoring assumption.

Method An alternative approach is explored for occupational epidemiology: competing risks and multistate model. In multistate models, subjects may contribute at-risk person-time by transitioning to multiple, sometimes competing, states. Subjects can transition to a state in which they are living with or die from the related disease (i.e., the competing risk of the outcome of interest). These models use a subdistribution hazard, in which competing events are accounted for in the survival probability and allow for additional baseline hazards for different states.

Results Competing risks and multistate models may allow researchers to build models that better reflect the biological complexity of an exposure contributing to multiple, related disease pathways simultaneously. For example, the exposure-response for the outcome of interest may be different if arriving at that outcome first through a related comorbidity than without that comorbidity.

Conclusions Although these models may improve our estimation, there are barriers to their implementation, including misclassification of disease, that will be discussed. 